\title{
A Novel Quantitative Index of Coronary Artery Motion from Multislice Computed Tomography and the Location of Coronary Artery Disease
}

\author{
Aiden JC O'Loughlin ${ }^{1, *}$, Linda Tang ${ }^{2}$, Daniel Moses ${ }^{2}$, Wisdom Tong DiagRad ${ }^{2}$, John K French ${ }^{3}$, David AB \\ Richards $^{3}$, A. Robert Denniss ${ }^{1,4}$, Annemarie Hennessy ${ }^{1}$ \\ ${ }^{1}$ School of Medicine, University of Western Sydney, Campbelltown, Sydney, 2560, NSW, Australia \\ ${ }^{2}$ Department of Radiology, Liverpool Public Hospital, Liverpool, Sydney, 2170, NSW, Australia \\ ${ }^{3}$ Department of Cardiology, Liverpool Public Hospital, Liverpool, Sydney, 2170, NSW, Australia \\ ${ }^{4}$ Department of Cardiology, Blacktown Public Hospital, Liverpool, Sydney, 2170, NSW, Australia \\ *Corresponding Author: A.O'Loughlin@uws.edu.au
}

Copyright (C) 2014 Horizon Research Publishing All rights reserved.

\begin{abstract}
Background: We describe a novel quantitative index of coronary artery motion (QCAM) from multislice computed tomography (MSCT) and test its association with the location of coronary artery disease. Methods: 25 patients with known or suspected coronary artery disease underwent ECG-gated MSCT. The coronary artery images were divided into 150 sections using landmarks that could be identified at time points at end-diastole and end-systole. QCAM was derived from the change in centerline length of the coronary sections between these time points. Plaques were identified and classified by type and severity of stenosis. Results: The mean QCAM was significantly less in the coronary sections with plaque $(94.3 \%+/-8.1 \%)$ than those without $(99.0 \%+/-10.2 \%) \quad(p=0.023)$. There was a significant correlation between QCAM and plaque stenosis (Spearman's rank correlation coefficient, $\rho=-0.192$, $\mathrm{p}=0.018$ ). The correlation between QCAM and plaque type approached statistical significance (Spearman's rank correlation coefficient, $\rho=-0.156, p=0.057)$. Sensitivity, specificity, positive and negative predictive values for the identification of coronary plaque within a section for QCAM $<100 \%$ were $80 \%, 46 \%, 27 \%$ and $90 \%$ respectively. Conclusions: QCAM is a novel quantitative measurement of coronary artery motion that correlates with the location of coronary artery disease. Quantitative evaluation of coronary artery motion provides a new approach to understanding the biomechanics of coronary artery disease.
\end{abstract}

Keywords MSCT, Coronary Artery Disease, Coronary Motion, Quantitative Modeling

\section{Introduction}

The response to endothelial injury model of atherosclerosis was first proposed by Virchow in 1856 [1] and revised by Ross and Glomset in 1973 [2-4]. The shear stress of coronary blood flow is the mechanism of injury most commonly implicated [5-10]. Local biomechanical injury due to the axial strain of cyclical coronary artery motion has also been shown to be an alternative mechanism of injury [11]. Coronary artery motion has been proposed to have an effect on the location of coronary artery disease by causing both low shear wall stress and increased wall strain [12]. We and other authors have previously shown that the compression pattern of coronary artery motion correlates with the location of coronary artery disease [13] and is predictive of the locations of culprit lesions in STEMIs [14-16].

Multislice computerized tomography (MSCT) is one of the most exciting recent developments in cardiology. Rapid advances in technology have resulted in MSCT entering 'prime-time' in recent years [17, 18]. Meta-analyses as early as 2007 support the potential use of 64 slice computed tomography coronary angiography in place of conventional coronary angiography (CCA) in carefully selected populations [19]. MSCT is superior to CCA alone in the analysis of plaque characteristics. Plaque characteristics have been shown to be different in patients with acute myocardial infarction or symptomatic stable angina pectoris [20] and to be predictive of future acute coronary syndromes [21]. MSCT readily allows quantitative evaluations to be performed. Coronary artery calcification is an example of a quantitative measurement that currently contributes significantly to risk stratification especially in persons with intermediate risk assessed by conventional risk analysis [22].

\section{Aim}

We describe a novel quantitative index of coronary artery motion (QCAM) obtained from MSCT. We test whether 
there is an association between QCAM and the location of coronary artery disease.

\section{Methods}

\subsection{Ethical Approval of the Study Protocol}

This study complied with the Declaration of Helsinki. The study was approved by the human research ethics committee of Royal Prince Alfred Hospital.

\subsection{Patients}

25 patients with known or suspected coronary artery disease who had undergone electrocardiographically-gated MSCT at Liverpool Hospital between April 2007 and January 2011 were evaluated. MSCT was performed using a retrospectively gated protocol on a 64 slice dual source cardiac CT scanner (SOMATON Definition, Siemens Healthcare). Patients were examined in a supine position, images extended from base of neck to the diaphragm during a single breath hold. Imaging parameters were as follows: detector collimation of $32 \times 0.6 \mathrm{~mm}$, slice acquisition $64 \times 0.6$ $\mathrm{mm}$ by means of a z-flying focal spot, gantry rotation time $330 \mathrm{~ms}$, pitch of $0.2-0.43$ adapted to the heart rate, tube current $400 \mathrm{mAs}$ per rotation, and tube potential $120 \mathrm{kV}$. The patients were randomly retrospectively identified from a database of MSCT studies performed during this period. Clinical data of the patients and MSCT results was obtained from MSCT reports and medical records.

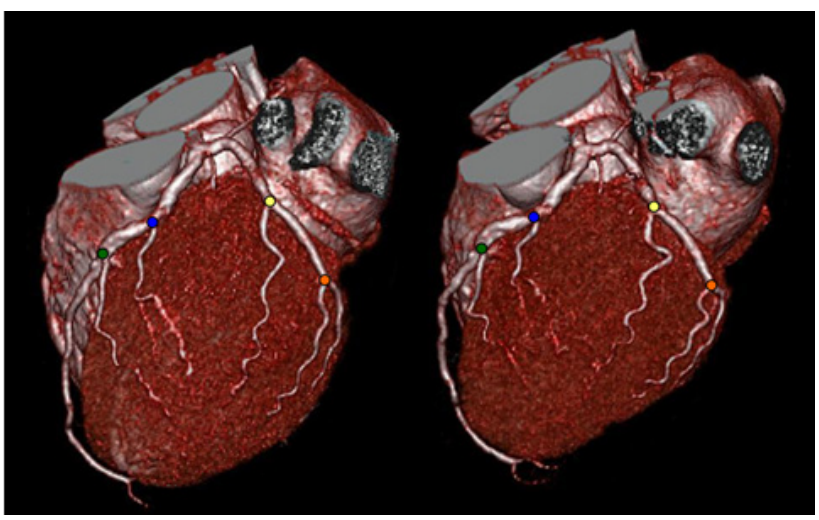

Figure 1. Vessel reconstructions at two time points in the cardiac cycle. Landmarks for the left anterior descending and left circumflex coronary arteries are shown with matching colors in the reconstructions

\subsection{Calculation of QCAM}

The images were reconstructed and manipulated on a dedicated cardiac work station. Raw data was reconstructed in $5 \%$ intervals throughout the cardiac cycle (which was defined as starting at the $\mathrm{R}$ wave). Vessel reconstructions that were representative of end-systole and end-diastole of the left ventricle were used for further analysis. The percent of the cardiac cycle for these two time points was recorded.
The coronary arteries were divided into sections using landmarks that could be identified at both time points. Most landmarks were bifurcations. Figure 1 shows the concepts of vessel reconstructions at end-systole and end-diastole and landmarks. Centerline lengths from the ostium of the vessel to the identified landmarks were measured on curved multiplanar reconstructions. The centerline lengths for each section were derived (Section $X=($ Ostium to Landmark $X)$ (Ostium to Landmark (X-1)). QCAM was defined as the percent shortening of centerline lengths between the time points (QCAM $=100 x$ (centerline length for section $X$ at end-systole)/(centerline length for section $\mathrm{X}$ at end-diastole) $\%$.

\subsection{Plaque Stenosis and Plaque Type Evaluation}

Plaques within coronary sections were identified. Plaque stenoses were classified by the maximal luminal diameter stenosis seen on any plane and the severity of stenosis was graded as none, non-obstructive $(<=50 \%)$ and significant $(>50 \%)$. Plaque types were classified as calcified arterial plaques (CAP) when calcified tissue occupied $>50 \%$ of the plaque area, plaques with $<50 \%$ calcium as mixed calcified plaques (MCAP), and plaques without any calcium as non-calcified plaques (NCAP), as previously described [18].

\subsection{Statistical Analyses}

Statistical analyses were performed using Stata version 8.0 (College Station, TX). Numerical values are expressed as mean and standard deviation unless otherwise stated. p-values $<0.05$ were considered statistically significant. The distributions of QCAM for sections with and without plaque were tested with an unpaired t test. Spearman's rank correlation coefficients were calculated for QCAM and both plaque stenoses and plaque types. Receiver operating characteristics (ROC) analysis was used to test the relationship between sensitivity and specificity at different cutoff values of QCAM.

\section{Results}

\subsection{Clinical and MSCT Characteristics of the Patients}

Baseline clinical characteristics of the patients are shown in Table 1. The age of the patients was $53.3(+/-15.6)$ years. $16(64 \%)$ of the patients were male. MSCT details are shown in Table 2. Chest pain was the indication for the majority of examinations. The elevated heart rate and high radiation doses reflect the selective use of retrospectively gated examinations in this study. 30 coronary plaques were identified in 11 patients. 4 patients underwent subsequent conventional coronary angiography (CCA). 2 of the patients had CCA within one week of their MSCT examinations. 1 of these patients was then referred for coronary artery bypass surgery. 1 was managed medically. 1 patient had CCA 6 
months later with percutaneous treatment of a left anterior descending stenosis (in a section reported as non-diagnostic on the MSCT examination due to heavy calcification). 1 patient had CCA 12 months later with only minor coronary disease identified.

Table 1. Baseline clinical characteristics

\begin{tabular}{|c|c|}
\hline Characteristic & \\
\hline Age in years (+/-standard deviation) & $53.3(+/-15.6)$ \\
\hline Male sex, number (\%) & $16(64 \%)$ \\
\hline Clinical information, & Number (\%) \\
\hline Family history of premature coronary disease & $5(20 \%)$ \\
\hline Hypercholesterolemia & $16(64 \%)$ \\
\hline History of Smoking & $7(28 \%)$ \\
\hline Diabetes & $8(32 \%)$ \\
\hline Hypertension & $14(56 \%)$ \\
\hline Systolic blood pressure $(\mathrm{mmHg})$ & $138(24 \%)$ \\
\hline Obesity & $10(40 \%)$ \\
\hline
\end{tabular}

Table 2. MSCT details

\begin{tabular}{|c|c|}
\hline Detail & Number \\
\hline Indication, & 19 \\
\hline Chest pain & 3 \\
\hline Cardiomyopathy & 1 \\
\hline Cardiac arrest & 1 \\
\hline Congenital heart disease & 1 \\
\hline Anomalous coronary artery & $74.9(+/-13)$ \\
\hline Heart Rate in beats per minute (standard \\
deviation) & $734(+/-300)$ \\
\hline Dose length product (standard deviation) & Value (standard deviation) \\
\hline Calcium scores & $89.9(+/-279)$ \\
\hline Agatston score & $71.4(+/-225)$ \\
\hline Volume score & $17.8(+/-56)$ \\
\hline Mass in mgCaHA &
\end{tabular}

\subsection{QCAM and Coronary Plaque}

Vessel reconstructions that were most representative of end-systole occurred at 37.1(+/- standard deviation of 5.1) \% of the cardiac cycle. Vessel reconstructions that were most representative of end-diastole occurred at $73.2(+/ 1$ standard deviation of 7.3 ) $\%$ of the cardiac cycle. Frequency distributions of QCAM for coronary sections without and with plaque are shown in Figure 2 with further categorization by plaque stenosis and plaque type in Table 3. QCAM was significantly less for coronary sections with plaque $(94.3 \%$ $+/-8.1 \%)$ than for those without plaque $(98.9 \%+/-8.1 \%)$ $(\mathrm{p}=0.023)$. There was a significant correlation between QCAM and plaque stenosis (Spearman's rank correlation coefficient, $\rho=-0.192, p=0.018)$. The correlation between QCAM and plaque type approached statistical significance (Spearman's rank correlation coefficient, $\rho=-0.156$, $\mathrm{p}=0.057$ ). Sensitivity, specificity, positive and negative predictive values for the identification of coronary plaque within a section for QCAM $<100 \%$ were $80 \%, 46 \%, 27 \%$ and $90 \%$ respectively.

Table 3. QCAM categorized by plaque stenosis and plaque type

\begin{tabular}{|c|c|c|c|}
\hline Categorization & Sections & $\begin{array}{c}\text { QCAM (+/- } \\
\text { standard } \\
\text { deviation) } \\
\end{array}$ & $P$ value \\
\hline & Number & & \\
\hline Plaque & & & $0.023^{\ddagger}$ \\
\hline None & 122 & $99.0(+/-10.2)$ & \\
\hline Present & 30 & $94.3(+/-8.1)$ & \\
\hline Plaque Stenosis & & & $0.018^{\dagger}$ \\
\hline None & 122 & $99.0(+/-10.2)$ & \\
\hline NonObstructive & 25 & $94.1(+/-7.4)$ & \\
\hline Significant & 5 & $95.2(+/-11.9)$ & \\
\hline Plaque Type & & & $0.057^{\dagger}$ \\
\hline None & 122 & $99.0(+/-10.2)$ & \\
\hline Non-calcified & 10 & $95.1(+/-9.1)$ & \\
\hline Mixed calcified & 5 & $89.3(+/-9.6)$ & \\
\hline Calcified & 15 & $96.8(+/-7.9)$ & \\
\hline
\end{tabular}

${ }^{\dagger}$ Unpaired $\mathrm{t}$ test ${ }^{\dagger}$ spearman's rank correlation

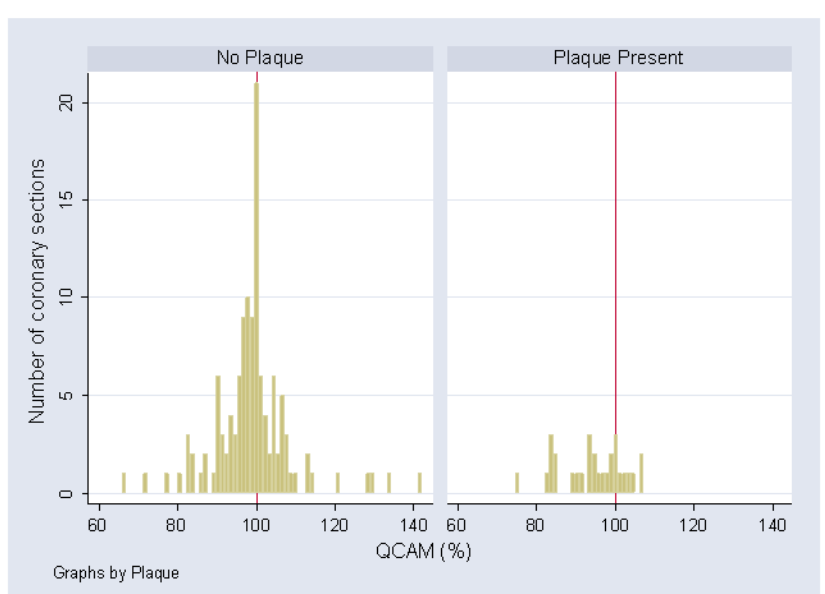

Figure 2. Frequency distribution of QCAM for coronary sections without and with plaque

\section{Discussion}

In this study, QCAM, a quantitative index of coronary motion from MSCT, is described for the first time. The mean 
QCAM was $4.7 \%$ less in coronary sections with coronary plaque than in those without plaque. This difference was statistically significant. Interestingly, the point estimates for QCAM were similar for both non-obstructive plaque and for plaque that caused significant stenosis. In terms of plaque type, the point estimate for QCAM was smallest for mixed calcific plaque and similar for non-calcified and calcified plaque. The small number of sections with mixed calcific plaque and the wide confidence interval for the point estimates preclude any further interpretation of this observation.

The frequency distribution of QCAM for coronary sections without and with coronary plaque (Figure 2) show considerable overlap of QCAM. In particular, a number of sections without plaque had QCAM values in the same range seen for sections with plaque. This is also reflected in the receiver operation characteristics of QCAM. Although the sensitivity $(80 \%)$ and negative predictive value $(90 \%)$ of a QCAM value of $<100 \%$ for detection of coronary plaque within a section were excellent, the specificity $(40 \%)$ and positive predictive value $(27 \%)$ were disappointing.

QCAM measures change in centerline length of a section of the coronary artery during the cardiac cycle. The decision to use this as a quantitative measure of coronary artery motion was based on the results of previous qualitative evaluations of coronary artery motion. In particular, change in centerline length was thought to best mirror the compression type of qualitative motion, which has previously been shown to correlate with the location of coronary artery disease [13]. The results in this study extend this finding to a quantitative method using MSCT. The compression pattern of coronary artery motion has also been shown to be predictive of the locations of culprit lesions in ST-segment elevation myocardial infarctions [14-16]. Whether QCAM is predictive of the location of culprit lesions in STEMIs is a clinically interesting hypothesis that remains to be tested.

This study has a number of limitations. Firstly, the use of landmarks to calculate QCAM results in averaging of the index over the artery section. This averaged index was then correlated with the coronary plaque. Plaque location was often focal and not involving the entire section for which QCAM was correlated. Secondly, this study uses retrospectively ECG-gated MSCTs. The mean heart rate of the examinations was 75 beats per minute. Most MSCTs currently performed use prospective ECG-gating, with acquisition of data predominantly within a pre-specified window in diastole. This precludes the measurement of QCAM as described in this study. Clinical benefit of QCAM measurement is required before the use of extra radiation to acquire the additional data for its calculation would be warranted.

\section{Conclusion}

QCAM is a novel measure which correlates with the location of coronary artery disease. Quantitative evaluation of coronary artery motion using MSCT provides an opportunity to better understand the biomechanics of coronary artery disease.

\section{Acknowledgements}

Ms Linda Tang was supported by a Summer Research Scholarship from the University of Western Sydney.

\section{Competing Interests}

The authors declare that they have no competing interests.

\section{REFERENCES}

[1] Topol E (Ed.). Textbook of Cardiovascular Medicine, 2nd edition: Lippincott Williams and Wilkins; 2002.

[2] Ross R, Glomset JA: Atherosclerosis and the arterial smooth muscle cell: Proliferation of smooth muscle is a key event in the genesis of the lesions of atherosclerosis. Science 1973, 180:1332-1339.

[3] Ross R: The pathogenesis of atherosclerosis--an update. N Engl J Med 1986, 314:488-500.

[4] Ross R: The pathogenesis of atherosclerosis: a perspective for the 1990s. Nature 1993, 362:801-809.

[5] Yoshida Y YT, Caro CG, Glagov S, Nerem RM, (Ed.). Role of blood flow in atherogenesis. Tokyo: Springer; 1988.

[6] Giddens DP, Zarins CK, Glagov S: The role of fluid mechanics in the localization and detection of atherosclerosis. J Biomech Eng 1993, 115:588-594.

[7] Krams R, Wentzel JJ, Oomen JA, Vinke R, Schuurbiers JC, de Feyter PJ, Serruys PW, Slager CJ: Evaluation of endothelial shear stress and 3D geometry as factors determining the development of atherosclerosis and remodeling in human coronary arteries in vivo. Combining 3D reconstruction from angiography and IVUS (ANGUS) with computational fluid dynamics. Arterioscler Thromb Vasc Biol 1997, 17:2061-2065.

[8] Feldman CL, Ilegbusi OJ, Hu Z, Nesto R, Waxman S, Stone PH: Determination of in vivo velocity and endothelial shear stress patterns with phasic flow in human coronary arteries: a methodology to predict progression of coronary atherosclerosis. Am Heart J 2002, 143:931-939.

[9] Coskun AU, Yeghiazarians Y, Kinlay S, Clark ME, Ilegbusi OJ, Wahle A, Sonka M, Popma JJ, Kuntz RE, Feldman CL, Stone PH: Reproducibility of coronary lumen, plaque, and vessel wall reconstruction and of endothelial shear stress measurements in vivo in humans. Catheter Cardiovasc Interv 2003, 60:67-78.

[10] Boutsianis E, Dave H, Frauenfelder T, Poulikakos D, Wildermuth S, Turina M, Ventikos Y, Zund G: Computational simulation of intracoronary flow based on 
real coronary geometry. Eur J Cardiothorac Surg 2004, $26: 248-256$.

[11] Zhu H, Friedman MH: Relationship Between the Dynamic Geometry and Wall Thickness of a Human Coronary Artery. Arteriosclerosis, Thrombosis, and Vascular Biology 2003, 23:2260-2265.

[12] John LCH: Biomechanics of Coronary Artery and Bypass Graft Disease: Potential New Approaches. Annals of Thoracic Surgery 2009, 87.

[13] Konta T, Bett JH: Patterns of coronary artery movement and the development of coronary atherosclerosis. Circ J 2003, $67: 846-850$

[14] O'Loughlin AJC, Byth K: The Stretch-Compression Type of Coronary Artery Movement Predicts the Location of Culprit Lesions Responsible for ST-Segment Elevation Myocardial Infarctions. Heart Lung and Circulation 2007, 16:265-268.

[15] Chan KH, Chawantanpipat C, Gattorna T, Chantadansuwan T, Kirby A, Madden A, Keech A, Ng MK: The relationship between coronary stenosis severity and compression type coronary artery movement in acute myocardial infarction. Am Heart J 2010, 159:584-592.

[16] O'Loughlin AJ, Byth K, French JK, Richards DA, Hennessy A, Denniss AR, Kovoor P: The compression type of coronary artery motion in patients with ST-segment elevation acute myocardial infarction and normal controls: a case-control study. BMC Res Notes 2011, 4:51.

[17] Budoff MJ, Dowe D, Jollis JG, Gitter M, Sutherland J, Halamert E, Scherer M, Bellinger R, Martin A, Benton R, et al: Diagnostic performance of 64-multidetector row coronary computed tomographic angiography for evaluation of coronary artery stenosis in individuals without known coronary artery disease: results from the prospective multicenter ACCURACY (Assessment by Coronary Computed Tomographic Angiography of Individuals Undergoing Invasive Coronary Angiography) trial. J Am Coll Cardiol 2008, 52:1724-1732.

Stein PD, Yaekoub AY, Matta F, Sostman HD: 64-slice CT for diagnosis of coronary artery disease: a systematic review. Am J Med 2008, 121:715-725.

[18] Abdulla J, Abildstrom SZ, Gotzsche O, Christensen E, Kober L, Torp-Pedersen C: 64-Multislice detector computed tomography coronary angiography as potential alternative to conventional coronary angiography: A systematic review and meta-analysis. European Heart Journal 2007, 28:3042-3050.

[19] Hammer-Hansen S, Kofoed KF, Kelbæk H, Kristensen T, Kühl JT, Thune JJ, Køber L: Volumetric evaluation of coronary plaque in patients presenting with acute myocardial infarction or stable angina pectoris-a multislice computerized tomography study. American Heart Journal 2009, 157:481-487.

[20] Motoyama S, Sarai M, Harigaya H, Anno H, Inoue K, Hara T, Naruse H, Ishii J, Hishida H, Wong ND, et al: Computed tomographic angiography characteristics of atherosclerotic plaques subsequently resulting in acute coronary syndrome. J Am Coll Cardiol 2009, 54:49-57.

[21] Rasmussen T, Zacho M, Kourmaeva D, Køber L, Kofoed KF: Koronararteriecalciumscore ved hjertecomputertomografi øger den prognostiske information hos udvalgte personer. Ugeskrift for laeger 2011, 173:2190-2195. 